\title{
DDX10 promotes the proliferation and metastasis of colorectal cancer cells via splicing RPL35
}

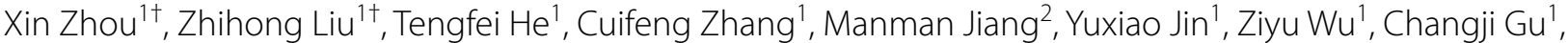 \\ Wei Zhang ${ }^{3^{*}}$ and Xiaodong Yang ${ }^{1 *}$ (D)
}

\begin{abstract}
Background: Colorectal cancer (CRC) has become the second deadliest cancer in the world and severely threatens human health. An increasing number of studies have focused on the role of the RNA helicase DEAD-box (DDX) family in CRC. However, the mechanism of DDX10 in CRC has not been elucidated.

Methods: In our study, we analysed the expression data of CRC samples from the Gene Expression Omnibus (GEO) and The Cancer Genome Atlas (TCGA) databases. Subsequently, we performed cytological experiments and animal experiments to explore the role of DDX10 in CRC cells. Furthermore, we performed Gene Ontology (GO)/Kyoto Encyclopedia of Genes and Genomes (KEGG) enrichment analysis and protein-protein interaction (PPI) network analyses. Finally, we predicted the interacting protein of DDX10 by LC-MS/MS and verified it by coimmunoprecipitation (Co-IP) and $\mathrm{qPCR}$.

Results: In the present study, we identified that DDX10 mRNA was extremely highly expressed in CRC tissues compared with normal colon tissues in the TCGA and GEO databases. The protein expression of DDX10 was measured by immunochemistry (IHC) in 17 CRC patients. The biological roles of DDX10 were explored via cell and molecular biology experiments in vitro and in vivo and cell cycle assays. We found that DDX10 knockdown markedly reduced CRC cell proliferation, migration and invasion. Then, we constructed a PPI network with the Search Tool for the Retrieval of Interacting Genes/Proteins (STRING). GO and KEGG enrichment analysis and gene set enrichment analysis (GSEA) showed that DDX10 was closely related to RNA splicing and E2F targets. Using LC-MS/MS and Co-IP assays, we discovered that RPL35 is the interacting protein of DDX10. In addition, we hypothesize that RPL35 is related to the E2F pathway and the immune response in CRC.
\end{abstract}

Conclusions: In conclusion, provides a better understanding of the molecular mechanisms of DDX10 in CRC and provides a potential biomarker for the diagnosis and treatment of CRC.

Keywords: Colorectal cancer, DEAD-box helicase 10 (DDX10), Oncogene, RPL35, mRNA splicing

\footnotetext{
*Correspondence: zhangweiszjs@163.com; 20194233020@stu.suda.edu.cn

${ }^{\dagger}$ Xin Zhou and Zhihong Liu are co-first authors

${ }^{1}$ Department of General Surgery, The Second Affiliated Hospital

of Soochow University, 1055 Sanxiang Road, Suzhou 215004, Jiangsu, China

${ }^{3}$ Department of Radiotherapy, The Second Affiliated Hospital of Soochow University, 1055 Sanxiang Road, Suzhou 215004, Jiangsu, China

Full list of author information is available at the end of the article
}

\section{Introduction}

Colorectal cancer (CRC) is the most common and second most deadliest malignant tumour of the digestive system worldwide [1]. Every year, patients newly diagnosed with CRC account for $10 \%$ of newly diagnosed cancer patients worldwide. Similarly, CRC accounts for $10 \%$ of cancerrelated deaths [1]. In the past 20 years, with the progress

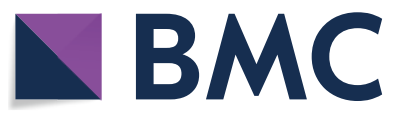

(c) The Author(s) 2022. Open Access This article is licensed under a Creative Commons Attribution 4.0 International License, which permits use, sharing, adaptation, distribution and reproduction in any medium or format, as long as you give appropriate credit to the original author(s) and the source, provide a link to the Creative Commons licence, and indicate if changes were made. The images or other third party material in this article are included in the article's Creative Commons licence, unless indicated otherwise in a credit line to the material. If material is not included in the article's Creative Commons licence and your intended use is not permitted by statutory regulation or exceeds the permitted use, you will need to obtain permission directly from the copyright holder. To view a copy of this licence, visit http://creativecommons.org/licenses/by/4.0/. The Creative Commons Public Domain Dedication waiver (http://creativeco mmons.org/publicdomain/zero/1.0/) applies to the data made available in this article, unless otherwise stated in a credit line to the data. 
of imaging technology and treatment strategies, the fiveyear survival rate of CRC has risen to $65 \%$. However, the situations of patients with advanced CRC remain dismal. The two-year survival rate of patients with advanced CRC is only approximately $20-30 \%$. Tumour metastasis is still the main cause of death for patients with this condition $[2,3]$. Therefore, reducing the proliferation and development of CRC cells has always been a difficult problem for medical workers.

In the past, research on the mechanism of tumour invasion and metastasis was mainly performed at the tissue and cellular levels. However, thanks to the rapid development of microarray and RNA sequencing (RNA-seq) technology, we began to carry out cancer research at the RNA level. In recent years, RNA helicases have attracted increasing attention from scientists. RNA helicases are enzymes that hydrolyse ATP to dissociate it from RNA and are mainly involved in RNA maturation, splicing and nuclear export processes [4]. Depending on their function and mechanism, RNA helicases are divided into different families. The RNA helicase DEAD-box (DDX) family belongs to superfamily 2 , which is named because of its highly conserved amino acid sequence (Asp-GluAla-Asp/His) [5]. There is a conserved core domain among DDX family members. This domain contains nine conserved motifs, including the most typical DEAD motif. These motifs endow DDX family members with ATP metabolism and RNA unwinding activities, which makes them RNA helicases. Many studies have proven that DDX family members play a crucial role in almost all steps of mRNA formation and translation [6]. Moreover, an increasing number of studies have proven that members of the DDX family are closely related to the proliferation and metastasis of tumour cells. For instance, DDX1 can affect the occurrence and metastasis of breast cancer, cervical cancer and colorectal cancer [7-11]. DDX5 is related to the progression of different tumours, such as breast cancer, colon cancer and multiple myeloma [1220]. DDX10 promotes the proliferation of breast cancer, osteosarcoma and ovarian cancer cells [21-23]. Interestingly, we found that many members of the DDX family affect the invasion and metastasis of CRC [11, 24-28], but no study has focused on the effect of DDX10 on the prognosis of CRC. Because the structure and function of DDX family members are similar, we speculated that DDX10 is a key factor affecting the prognosis of CRC.

In our study, we analysed the expression data of CRC samples from the Gene Expression Omnibus (GEO) and The Cancer Genome Atlas (TCGA) databases to determine whether the mRNA expression of DDX10 in CRC tissues is higher than that in normal tissues. Moreover, the clinical samples we collected from The Second Affiliated Hospital of Soochow University were used for immunohistochemistry (IHC), which could detect the differences in DDX10 expression at the protein level. Subsequently, we performed cytological experiments and animal experiments to explore the role of DDX10 in CRC cells. Furthermore, we performed Gene Ontology (GO)/Kyoto Encyclopedia of Genes and Genomes (KEGG) enrichment analysis and protein-protein interaction (PPI) network analyses, which enabled us to better understand how DDX10 affects the invasion and metastasis of CRC. Finally, we predicted the interacting protein of DDX10 by LC-MS/MS and verified it by Co-IP and qPCR.

\section{Materials and methods}

Patients and specimens

Between January and December 2014, we collected 35 pairs of cancer and paracarcinoma tissues from patients with CRC at the Gastrointestinal Surgery Department of the Second Affiliated Hospital of Soochow University (Jiangsu Province, China). Our study was approved by the ethics committee of Soochow University. We obtained informed consent from every patient. All specimens were stored at $-80^{\circ} \mathrm{C}$.

\section{Immunohistochemistry}

Colorectal cancer and paracarcinoma tissues were dewaxed in xylene and rehydrated through different gradients of alcohol. Then, we placed paraffin in $3 \% \mathrm{H}_{2} \mathrm{O}_{2}$ for $15 \mathrm{~min}$ at $22^{\circ} \mathrm{C}$. Next, the slide was heated in citrate buffer. After washing several times with PBS ( $\mathrm{pH}=7.2$ ), the slide was placed into the solution with a primary antibody against DDX10 (dilution 1:50, 17857-1-AP, Proteintech, USA) at $4{ }^{\circ} \mathrm{C}$ for more than $12 \mathrm{~h}$. Then, we added secondary antibody at $22{ }^{\circ} \mathrm{C}$ for $10 \mathrm{~min}$. Finally, the slide was stained after the addition of 3,3'-diaminobenzidine (DAB) solution.

\section{Differential expression analysis}

The TCGA is an open public tumour information database. We selected 50 pieces of CRC data, including that for cancer and paracarcinoma tissues, from the TCGA database to compare the mRNA expression of DDX10. The GEO database (http://www.ncbi.nlm.nih. gov/geo) [29] is an international public database that stores abundant high-throughput functional genomics data. The gene expression dataset analysed in our study was GSE74604 downloaded from the GEO. GSE74604, based on the GPL6104 platform (Illumina humanRef-8 v2.0 expression beadchip), contained data for 30 paired normal and tumour colorectal samples. We analysed the differences in gene expression data for 60 samples. Then, we also used Gene Expression Profiling Interactive Analysis 2 (GEPIA2, http://gepia.cancer-pku.cn/) [30] and 
ONCOMINE (https://www.oncomine.org/) to verify the results. GEPIA2 is a web tool that includes RNA-seq data for a large number of tumour and normal samples from the TCGA and Genotype-Tissue Expression (GTEx) projects. Gene expression data from ONCOMINE are freely available to the public. ONCOMINE is meant to facilitate related studies by providing genome-wide expression array data.

\section{Real-time PCR}

Total RNA was extracted by SuPerfecTRI ${ }^{\mathrm{TM}}$ (Cat\#3101100) (Shanghai Pufei Biotech Co., Ltd). This RNA was then reverse-transcribed to cDNA by M-MLV Reverse Transcriptase (M1705) (Promega, Beijing, China). SYBR Master Mixture (TAKARA, Tokyo, JP) was used for real-time PCR. We used the expression of endogenous GAPDH as a control to take standardized quantification. The DDX10 primer sequences were as follows: forward, $5^{\prime}$-TTGAGGTTCTCCGAAAAGTAGG- ${ }^{\prime}$ and reverse, $5^{\prime}$-ACATTTGGAGGTCGGTAGCAT- $3^{\prime}$; the GAPDH primer sequences were as follows: forward, $5^{\prime}$-TGACTT CAACAGCGACACCCA-3' and reverse, 5'-CCATTG CCCGTGTTCTCACA-3'; the $60 \mathrm{~S}$ ribosomal protein L35 (RPL35) primer sequences were as follows: forward, 5'-TGACTTCAACAGCGACACCCA-3' and reverse, 5'-GCTCCTTCCGCTGCTGCTTC-3'.

\section{Cell lines and lentiviral transduction}

Our study used the human CRC cell lines RKO, HCT116, SW480 and LoVo, which were purchased from GeneChem (Shanghai, China). The cells were cultured in Dulbecco's modified Eagle's medium (DMEM; HyClone, Thermo Fisher Scientific) containing 10\% foetal bovine serum (FBS; Gibco-Invitrogen Corp.) at $37{ }^{\circ} \mathrm{C}$ with $5 \%$ $\mathrm{CO}_{2}$ in strict accordance with the standard. All lentiviruses used in this research were purchased from GeneChem (Shanghai, China). We transfected short hairpin RNA (shRNA) lentivirus targeting DDX10 and empty vector (controls) into HCT116 cells and RKO cells in strict accordance with the official technical instructions. The DDX10 RNAi sequence was 5'-GATGTGAGCAAG TTACCTATA-3'.

\section{Celigo cell growth assay}

HCT116 cells and RKO cells were plated in 96-well plates (1500 cells/well). Next, we cultured the cells at $37{ }^{\circ} \mathrm{C}$ with $5 \% \mathrm{CO}_{2}$. During the next five days, we counted the number of cells with a Celigo Imaging Cytometer (Nexcelom Bioscience, Lawrence, MA, USA) every day. All experiments were repeated at least three times.

\section{Colony formation assay and apoptosis assay}

First, we collected HCT116 cells and RKO cells. Next, those cells were added to complete medium (10\% FBS) and placed into 6-well plates (800 cells/well). Then, we cultured the cells in standard conditions for 8 days and changed the medium every three days. Then, they were fixed for 30-60 min with 4\% paraformaldehyde. Last, we added $1000 \mu \mathrm{l} /$ well crystal violet at room temperature. Approximately 10-20 min later, we counted the number of cells under a fluorescence microscope (Olympus).

We used annexin V-APC (eBioscience) to stain these cells to analyse apoptosis in strict accordance with official technical instructions. The cells were plated in 6-well plates $\left(5 \times 10^{5}\right.$ cells/well). Then, we collected the cells and washed them with PBS. After the cells were resuspended, we added $10 \mu \mathrm{l}$ annexin $\mathrm{V}$-APC to the suspensions. Finally, we cultured the cells at $22^{\circ} \mathrm{C}$ and protected them from sunlight. After $15 \mathrm{~min}$, the cells were sorted by flow cytometry (C6 PLUS, BD).

\section{MTT assay}

MTT assays were used to observe the proliferation of cells. All operations were in strict accordance with the standard. First, we placed HCT116 or RKO cells with DDX10 knockdown and negative control cells into 96-well plates ( 1500 cells/well) in triplicate. Then, $100 \mu \mathrm{l}$ medium was added to every well. Finally, a microplate reader (M2009PR, Tecan Infinite) was used to quantify the colour change $(490 \mathrm{~nm})$ at $24 \mathrm{~h}, 48 \mathrm{~h}, 72 \mathrm{~h}, 96 \mathrm{~h}$ and $120 \mathrm{~h}$.

\section{Wound-healing assay}

First, we harvested HCT116 cells and RKO cells (negative control and DDX10 knockdown). Then, the cells were cultured in 96-well plates. After the confluence reached $90 \%$, we aspirated the medium of every well and used PBS to wash the wells three times. Next, we used a pipette tip to gently draw a straight line down the wells of a 96-well plate. Finally, medium was added to every well after three washes with PBS. After $0 \mathrm{~h}, 24 \mathrm{~h}$ and $72 \mathrm{~h}$, we used the Celigo platform to scan the plate and analyse the migration area.

\section{Transwell migration and invasion assays}

Transwell assays were used to assess the migration and invasion abilities of HCT116 cells and RKO cells. HCT116 cells or RKO cells $\left(1 \times 10^{5}\right)$ in $100 \mu \mathrm{l}$ medium that had been cultured in serum-free medium were added to the upper chamber, and $600 \mu \mathrm{l}$ culture medium (30\% FBS) was added to the lower chamber. Then, the plate was incubated at $22{ }^{\circ} \mathrm{C}$ overnight. Finally, we dyed the cells on the lower surface of the membrane to analyse 
cell migration. The Transwell invasion assay was carried out as described above, but Matrigel was added to the bottom of the upper chamber.

\section{Animal studies}

The animal experiment of our study used 5-week-old female BALB/c nude mice (GemPharmatech, Co., Ltd., China) weighing between 16 and $19 \mathrm{~g}$. Twenty nude mice were randomly divided into two groups with 10 mice in each group. Two groups (negative control and DDX10 knockdown) of HCT116 cells (stably expressing luciferase) $\left(2 \times 10^{6}\right.$ cells $\left./ 100 \mu \mathrm{l}\right)$ were injected into the mice via the tail vein. Then, we used an in vivo imaging system (Lumina LT, Perkin Elmer, USA) to observe tumour metastasis every week. We anaesthetized the mice before performing imaging monitoring. First, the mice were intraperitoneally injected with D-luciferin $(15 \mathrm{mg} / \mathrm{ml})$ at a concentration of $10 \mu \mathrm{l} / \mathrm{g}$. After $15 \mathrm{~min}$, the mice were anaesthetized by intraperitoneal injection of $0.7 \%$ pentobarbital sodium at a concentration of $10 \mu \mathrm{l} / \mathrm{g}$. After a few minutes, the mice were anaesthetized and placed under bioluminescence imaging for imaging monitoring. All mice were cultured in a specific-pathogen-free (SPF) culture environment. At the same time, we also measured and recorded the tumour size.

\section{Mass spectrometry and protein identification}

Briefly, we used a scraper to harvest RKO cells that stably expressed Flag-DDX10 and lysed the cells with lysis buffer. The reference dosage for Co-IP was $400 \mu \mathrm{l}$ FLAG beads to collect $15,000 \mu \mathrm{g}$ of protein. First, we used weak lysis buffer to wash the beads four times. Then, we carried out SDS-PAGE and placed the gel into Coomassie brilliant blue R250 solution for staining. Next, we collected the protein bands and used LC-MS for analysis. The original graph file (.raw) exported from Q Exactive was changed into a ".mgf" file by Proteome Discoverer 2.1 (Thermo Fisher Scientific), and then we delivered these data to the MASCOT2.6 server for database searching by built-in facilities. Then, we received the search files (.dat) that had been processed from the MASCOT server. Finally, we selected candidate peptides according to the standard of FDR $<0.01$. The protein database used in this analysis was UniProt_HomoSapiens_20386_20180905.

\section{LinkedOmics}

LinkedOmics (http://www.linkedomics.org/) [31] is an unique platform for biologists and clinicians to access, analyze and compare cancer multi-omics data within and across tumor types. In our study, we used the "colorectal adenocarcinoma (TCGA_COADREAD)" dataset to analyse the correlation between E2F family and DDX10 or RPL35.

\section{Co-immunoprecipitation assay}

First, we collected RKO cells that overexpressed DDX10 after washing them three times with PBS. Then, these cells were added to precooled cell lysis buffer. Next, the final cell lysates were sonicated and centrifuged at 14,000 rpm for $10 \mathrm{~min}$. The lysates were mixed with preblocked Flag beads (A2220, Sigma) at $4{ }^{\circ} \mathrm{C}$ overnight. After Co-IP, the Flag beads were washed as follows. First, we washed with lysis buffer three times. Then, we added $6 \times$ loading buffer and left the samples at room temperature for $10 \mathrm{~min}$. Finally, the cell lysates were mixed with $5 \times$ sodium dodecyl sulfate (SDS) loading buffer and then boiled for $10 \mathrm{~min}$. Proteins were eluted from the beads and separated by western blotting.

\section{Western blot analysis}

We used a 15\% SDS-PAGE gel to isolate proteins, and the proteins were transferred to a PVDF membrane. The primary antibodies used in our study were mouse antiGAPDH (1:5000; Santa Cruz, USA), rabbit anti-RPL35 (1:100; ABclonal, China), rabbit anti-DDX10 (1:300; Proteintech, USA), mouse anti-FLAG (1:2,000; Sigma, USA), rabbit anti-RPL18 (1:100; ABclonal, China), rabbit antiHNRNPU (1:100; ABclonal, China), rabbit anti-NCL (1:100; ABclonal, China), rabbit anti-PRMT5 (1:100; ABclonal, China), rabbit anti-E2F1 (1:100; ABclonal, China), rabbit anti-E2F4 (1:100; ABclonal, China), rabbit anti-E2F8 (1:100; ABclonal, China), goat anti-rabbit IgG (1:2,000; CST, USA), and goat anti-mouse IgG $(1: 2,000$; CST, USA).

\section{Enrichment analyses and PPI network construction}

GO and KEGG analyses are important bioinformatics tools for annotating genes and researching gene functions, biological processes and signalling pathways [32-34]. In our study, we analysed the genes that were downregulated by DDX10 antibody using R version 4.0.5. The PPI network for these genes was predicted using the Search Tool for the Retrieval of Interacting Genes (STRING; https://string-db.org/) online database [35].

\section{Gene set enrichment analysis (GSEA)}

The RNA-seq data from the COAD and READ datasets were obtained from the TCGA portal (https://portal. gdc.cancer.gov/). Then, we divided the TCGA samples into two groups according to the expression of DDX10 and analysed these two groups of data by GSEA. Finally, we screened the results with the criterion of normalized enrichment score $(\mathrm{NES}) \geq 1.0$ and adjusted $\mathrm{P}$ value $<0.25$. 
Tumour immune estimation resource (TIMER) database analysis

TIMER (https://cistrome.shinyapps.io/timer/) [36, 37] is an interactive website offering comprehensive analysis of immune infiltration among different types of cancer. TIMER uses various immune deconvolution methods to calculate the immune infiltrate abundances. Moreover, the subscriber can obtain high-quality figures. In this work, we searched "RPL35; COAD" in the "gene module" and performed an analysis of immune infiltration.

\section{Statistical analysis}

We used Prism version 8.0 software (GraphPad Software Inc.) to analyse all the data in this study. Unpaired t tests were used to compare two groups, and one-way ANOVA was used to compare multiple groups. The screening criteria for all data was $\mathrm{P}$ value $<0.05$. All of the trials were repeated at least three times.

\section{Results}

DDX10 expression is upregulated in CRC tissue and CRC cell lines

We analysed the gene expression of 50 paired cancer and paracarcinoma tissues selected from the TCGA database and found that the mRNA expression of DDX10 in CRC tissues was evidently higher than that in paracarcinoma tissues (Fig. 1A). By analysing the mRNA expression data of 30 pairs of samples from the GEO database (GSE74604), we obtained a list of differentially expressed genes (Additional file 1: Fig. S1A); the mRNA expression of DDX10 in CRC was evidently higher than that in paracarcinoma tissues (Fig. 1A). Next, we used online analytics websites to retrieve data for DDX10. The results of the GEPIA2 and ONCOMINE database analysis also verified that the mRNA expression of DDX10 was significantly different between tumour and normal tissues (Additional file 1: Fig. S1B-F; Table 1). All these database results showed that the mRNA expression of DDX10 was upregulated in CRC tissue. As expected, compared with
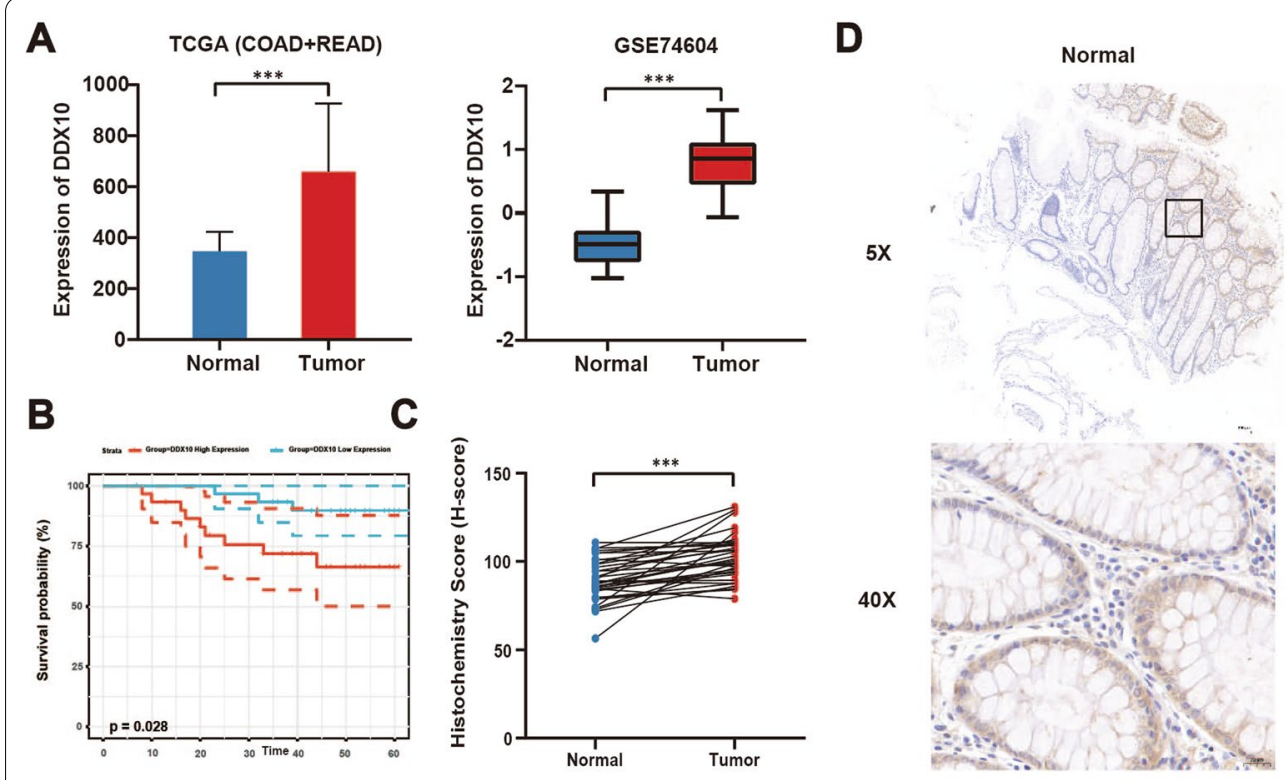

\section{C}
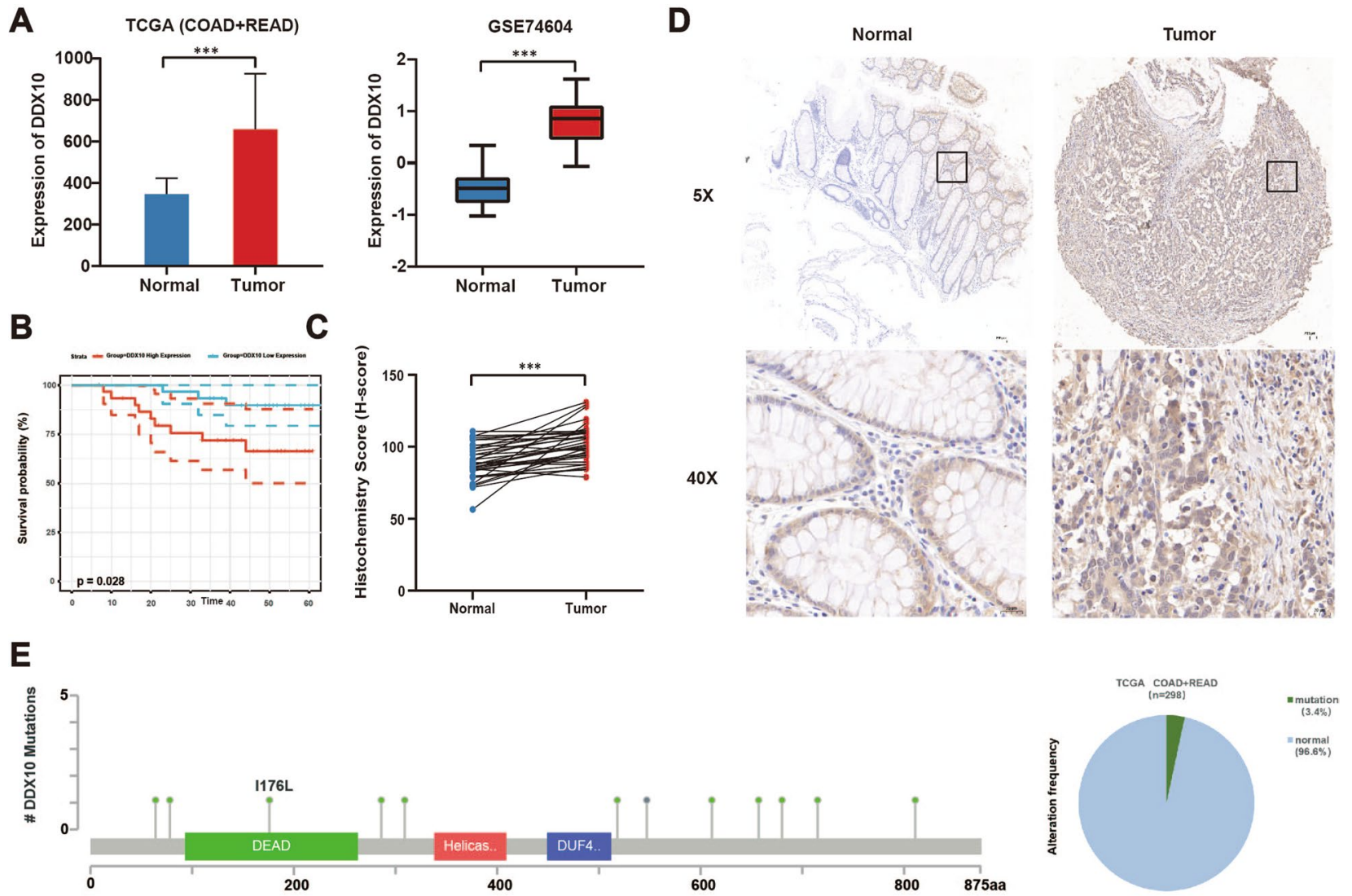

Fig. 1 Clinical significance of DDX10 expression in colorectal cancer (CRC). A mRNA expression of DDX10 in CRC tissues and normal colon tissues in The Cancer Genome Atlas (TCGA) database and Gene Expression Omnibus (GEO) database; B Kaplan-Meier disease-free survival curves of patients with CRC according to DDX10 expression in GSE17537; C Immunohistochemical results of clinical samples $(n=35, H$-score, $P<0.05)$; D Immunohistochemical staining for DDX10 in CRC tissues and normal tissues; E Positions and frequency of mutations in DDX10 among CRC cases in TCGA dataset. Number of mutations was observed in twelve cases and frequency was $3.4 \%$ 
Table 1 The expression levels of DDX10 in different types of tumor and normal tissues (UALCAN)

\begin{tabular}{|c|c|c|c|c|}
\hline Type of tumor & Maximum & Minimum & Median & $P$ value \\
\hline \multicolumn{5}{|l|}{ BLAC } \\
\hline Tumor & 26.716 & 1.093 & 12.897 & \multirow[t]{2}{*}{ 4.598200E-03 } \\
\hline Normal & 15.437 & 7.483 & 12.837 & \\
\hline \multicolumn{5}{|l|}{$\mathrm{CHOL}$} \\
\hline Tumor & 16.393 & 4.659 & 10.713 & \multirow[t]{2}{*}{$1.700750 \mathrm{E}-12$} \\
\hline Normal & 4.804 & 2.332 & 3.305 & \\
\hline \multicolumn{5}{|l|}{ COAD } \\
\hline Tumor & 33.192 & 7.078 & 18.391 & \multirow[t]{2}{*}{$1.624478 \mathrm{E}-12$} \\
\hline Normal & 11.336 & 3.544 & 7.439 & \\
\hline \multicolumn{5}{|l|}{ ESCA } \\
\hline Tumor & 38.759 & 4.748 & 16.977 & \multirow[t]{2}{*}{$5.969599 \mathrm{E}-07$} \\
\hline Normal & 15.079 & 4.143 & 6.562 & \\
\hline \multicolumn{5}{|l|}{ LIHC } \\
\hline Tumor & 15.539 & 0.842 & 6.313 & \multirow[t]{2}{*}{$1.624367 E-12$} \\
\hline Normal & 5.35 & 1.06 & 3.834 & \\
\hline \multicolumn{5}{|l|}{ READ } \\
\hline Tumor & 30.994 & 1.888 & 17.34 & \multirow[t]{2}{*}{ 1.086199E-08 } \\
\hline Normal & 10.149 & 7.718 & 8.57 & \\
\hline \multicolumn{5}{|l|}{ STAD } \\
\hline Tumor & 31.934 & 4.648 & 14.939 & \multirow[t]{2}{*}{$<1 \mathrm{E}-12$} \\
\hline Normal & 12.23 & 2.641 & 6.215 & \\
\hline
\end{tabular}

BLCA bladder urothelial carcinoma; STAD stomach adenocarcinoma; COAD colon adenocarcinoma; $R E A D$ rectum adenocarcinoma; $C H O L$ cholangiocarcinoma; LIHC liver hepatocellular carcinoma; ESCA esophageal carcinoma

that in paracarcinoma tissues, the protein expression of DDX10 in tumour tissues was significantly elevated (Fig. 1C, D). Survival analyses of disease-free survival in COAD revealed that the expression of DDX10 is associated with the prognosis of patients (Fig. 1B). The rate of mutations in DDX10 was $3.4 \%$ in the TCGA (Fig. 1E). Furthermore, compared with normal intestinal epithelial cells (NCM460), higher expression of DDX10 was shown in many CRC cell lines (LoVo, HCT116, RKO, SW480) with GAPDH as an internal reference gene (Fig. 2A).

\section{DDX10 promotes CRC cell proliferation and metastasis in vitro}

Analysis of the relationship between the mRNA expression of DDX10 and the staging of CRC in the ONCOMINE database indicated that DDX10 expression in tumour tissues in M1 stage (Additional file 1: Fig. S1G) or Dukes' D stage (Additional file 1: Fig. S1H) was higher than that in tissues in M0 or Dukes' A stage.

To clarify the effect of DDX10 on the invasion and metastasis of CRC cells, LV-shDDX10 HCT116 and RKO cells were generated, and the influence on DDX10 expression was proven by qRT-PCR (Additional file 2: Fig. S2A). MTT (Fig. 2B, C) and cell counting assays (Fig. 2D) showed that the decrease in DDX10 expression significantly suppressed the growth of HCT116 and RKO cells. Next, the results of the colony formation test revealed that the colony number of the shDDX10 cell group were evidently reduced compared with that of the shCtrl cell group (Fig. 2E). Then, we assessed cell apoptosis by fluorescence-activated cell sorting (FACS). Five days after shRNA lentivirus infection, the number of apoptotic HCT116 and RKO cells had increased markedly in the experimental group (shDDX10) (Fig. 2F). These outcomes suggested that HCT116 and RKO cell proliferation was suppressed due to the decreased expression of DDX10. Finally, the outcomes of the wound-healing assay and Transwell migration assay indicated that decreased expression of DDX10 could significantly reduce the wound-healing rate and the number of metastatic HCT116 and RKO cells (Fig. 2G, H). Transwell invasion assays also showed that, compared with control cells, fewer HCT116 and RKO cells with extremely low expression of DDX10 passed through the cell-permeable membrane (Fig. 2I). This result showed that DDX10 could enhance the invasiveness of CRC cells. Based on the above experimental results, our study indicates that DDX10 potentially has the ability to promote the development and metastasis of CRC.

\section{DDX10 promotes colon cancer cell metastasis in vivo}

To clarify the function of DDX10 in metastasis in vivo, we constructed a metastasis model of CRC in nude mice (Fig. 3A). In our study, colorectal cancer metastasis was found only in the lung. The number and weight of lung metastatic nodules in mice were observed 37 days after tail vein injection. The final results showed that the number of lung metastatic nodules was lower (Fig. 3C) (the metastasis rates of the negative control group and knockdown group were $90 \%$ and $50 \%$, respectively) (Fig. 3B), and the total weight of all metastatic nodules in mice injected with HCT116/LV-shDDX10 cells was higher than that of all metastatic nodules in mice injected with HCT116/LV-shCtrl cells (Fig. 3E, F). In addition, the total fluorescence intensity in the region was greater in mice that were injected with HCT116/LV-shDDX10 cells than in those injected with HCT116/LV-shCtrl cells (Fig. 3D). Therefore, this study demonstrated that DDX10 has roles in promoting CRC growth and progression in vivo.

\section{DDX10 expression is positively correlated with the cell cycle and RNA splicing}

The PPI network among these proteins interacting with DDX10 was predicted by STRING tools. We found 110 proteins interacting with DDX10 in the suspension of the Co-IP assay by LC-MS. These 110 proteins were involved in a complex PPI network with a total of 108 nodes and 


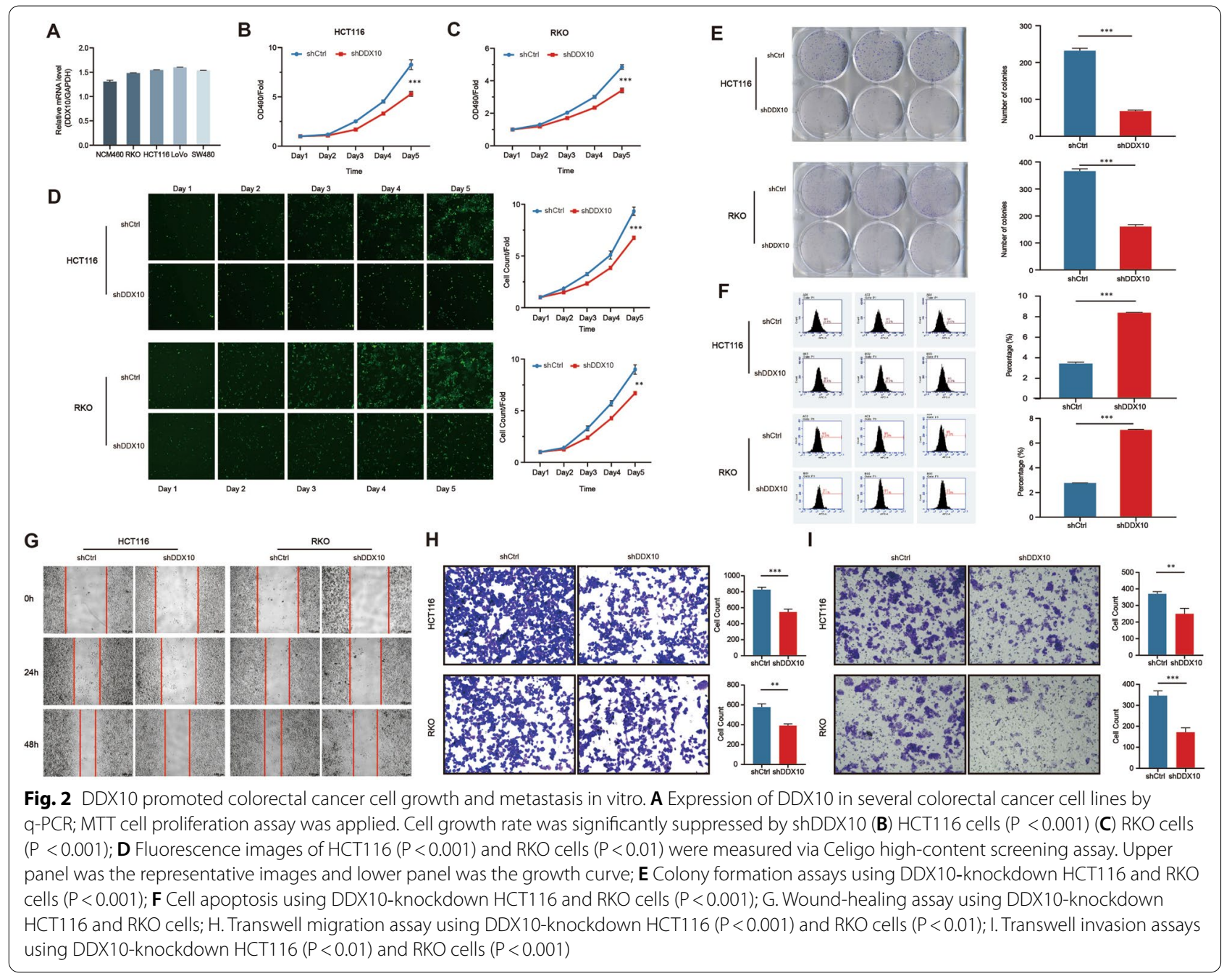

1,181 edges (Fig. 4A). In this network, the average local clustering coefficient was 0.649 , and the PPI enrichment $\mathrm{P}$ value was $<1.0 \mathrm{e}-16$.

To explore the mechanism by which DDX10 regulates the occurrence and development of CRC, these 110 proteins were used for GO/KEGG enrichment analysis. The results indicated that DDX10 was positively correlated with microtubule binding, the cell cycle and RNA splicing (Fig. 4B-E).

\section{DDX10 may alternatively splice the mRNA of RPL35 and then work through the E2F pathway}

To better study the biological functions and pathways in which DDX10 participates, we searched for distinctive proteins that interact with DDX10. Cell extracts were applied to LC-MS/MS analysis, and the output is shown in the Additional file 3: Table S1. A large number of Co-IP assays and western blot experiments were used to verify the interaction between the proteins selected by LC-MS/MS and DDX10 (Fig. 5A). Eventually, we found that RPL35 is very likely to interact with DDX10. As expected, the q-PCR and western blot results showed that the decreased expression of DDX10 obviously reduced the expression of RPL35, which verified our conclusion (Fig. 5B, C). At the same, we detected the expression of two mRNA isoforms of RPL35 in normal HCT116 cells and DDX10 knockdown HCT116 cells. Amazingly, the absence of DDX10 did change the two isoforms of RPL35 (Fig. 5D). E2F transcription factors (E2Fs) are the most important genes regulating the progression of CRC. The outcomes of LinkedOmics indicated that DDX10 and RPL35 are closely related to E2F1, E2F4 and E2F5 in CRC (Fig. 5E). In addition, from the GSEA results, we observed that the expression of DDX10 was closely related to E2F targets (Fig. 4E). Therefore, we use western blot to verify that E2Fs pathway is indeed the downstream pathway of DDX10 and RPL35 (Fig. 5F). Considering the above results, we propose that DDX10 
may regulate the occurrence and development of CRC through the E2F pathway after alternatively splicing RPL35 mRNA.

\section{DDX10 is most likely related to the immune response of CRC}

The TIMER results indicated that the expression of DDX10 is closely related to the infiltration of CD4+ T cells, CD8 + T cells and macrophages in COAD (Fig. 5G). Therefore, we surmised that DDX10 influences the occurrence and development of CRC by regulating immune cell infiltration, which would be an interesting research direction.

\section{Discussion}

In recent years, CRC has become the second leading cause of death in cancer patients. The ultimate cause of death in CRC patients is usually tumour metastasis. Therefore, it is very important to explore the mechanism underlying the metastasis of CRC cells. Many DDX family members, including DDX1, DDX3, DDX5, DDX17, DDX27 and DDX56, have been confirmed to be closely related to CRC [11, 24, 28, 38-40]. Moreover, through the research of Patrick Linder et al. [5] we know that the members of the DDX family are similar in structure and function. Hence, we reasonably speculated that DDX10 plays a key role in the invasion and metastasis of CRC cells.

DDX10 is a member of the DDX family that is characterized by the presence of an Asp-Glu-Ala-Asp (DEAD) motif, and its main function is to mediate the biogenesis of ribosomes [41]. However, it was not until the late 1990s that DDX10 attracted the attention of biologists [41, 42]. Our pancancer analysis results showed that DDX10 is highly expressed in a variety of cancer tissues; therefore, DDX10 is likely to be a key gene in many cancers. Previous studies have shown that the fusion of NUP98 and DDX10 may lead to leukaemia [43]. Through gene rearrangement studies, Dr. Jiao confirmed that DDX10 is a potential oncogene affecting the growth and proliferation of breast cancer cells [21]. Recently, it was confirmed that the expression of DDX10 is significantly higher in osteosarcoma patients [22]. Interestingly, DDX10 plays an antitumour role in the development of ovarian cancer. Studies have shown that decreased expression of DDX10 promotes the proliferation of ovarian cancer through the Akt/NF-kB pathway [23]. In our study, we first reported

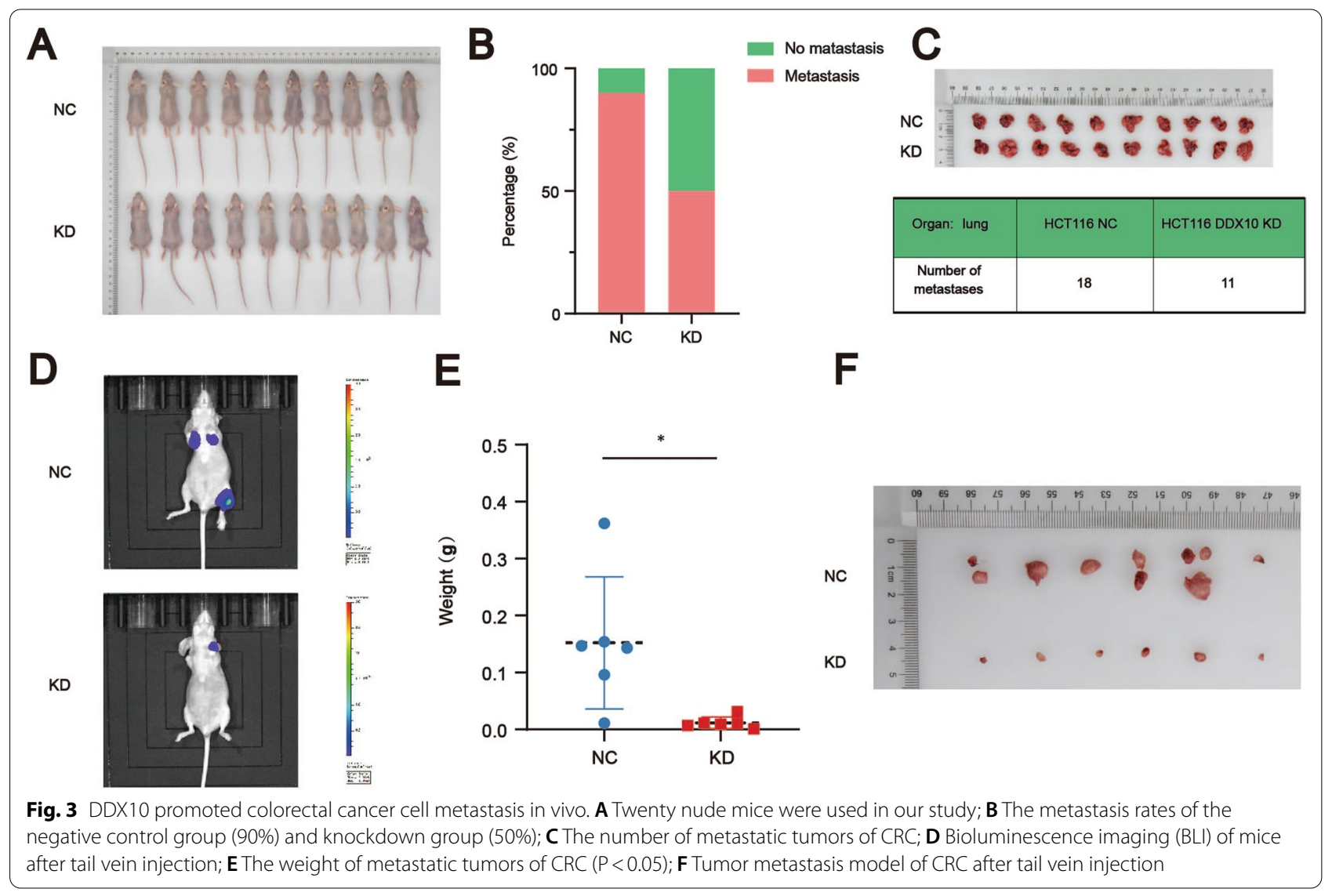




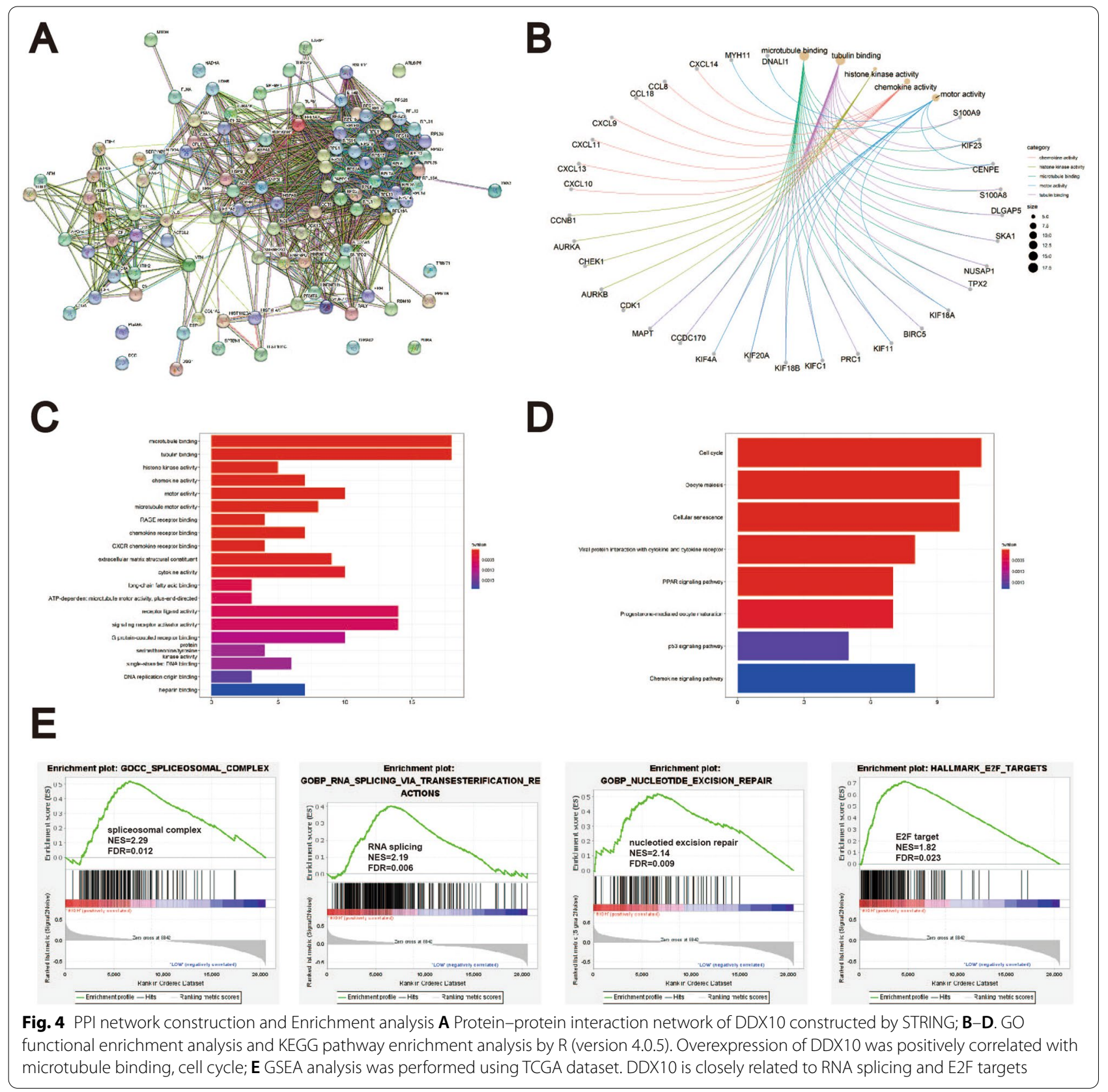

that DDX10 was upregulated in CRC and was closely associated with CRC clinical stage and prognosis. To the best of our knowledge, this is the first study on the function of DDX10 as a carcinogenic driver and prognostic biomarker for CRC.

In our study, the data from multiple databases were used to verify that the expression of DDX10 in tumour tissues was evidently higher than that in normal tissues, and the difference was also obvious in tumournode-metastasis (TNM) stage and Dukes' stage. Then, we used a lentiviral transfection method to knockdown and overexpress DDX10 to carry out cytological experiments and to construct a tumour metastasis model in mice, which helped us explore the effect of DDX10 on the growth, invasion and metastasis of CRC cells. These results showed that the growth of CRC cells was significantly inhibited after DDX10 knockdown and that the invasion ability was also significantly reduced. The decrease in the number and total weight of metastatic tumours in mice injected with shDDX10-CRC cells also fully demonstrated that the expression of DDX10 promoted the migration of CRC. The above results strongly 


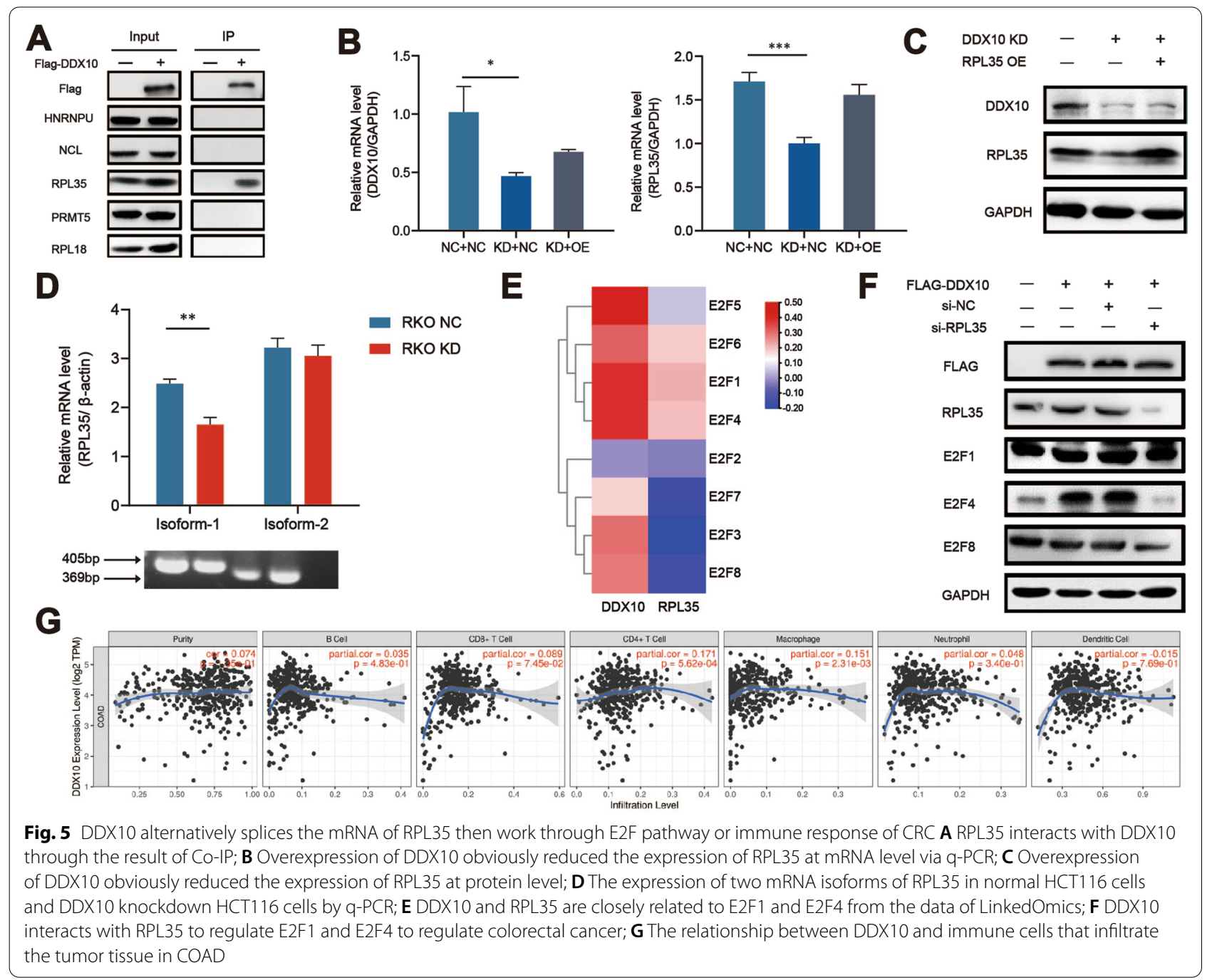

suggest that DDX10 is a key gene regulating the invasion and metastasis of CRC and that DDX10 could be used as a new target for CRC treatment.

To better comprehend the mechanism of DDX10 in CRC, we carried out LC-MS/MS analysis and employed Co-IP, q-PCR and western blotting to verify the interaction. Fortunately, we found that DDX10 and RPL35 were strongly coexpressed. RPL35 encodes a ribosomal protein that is a component of the $60 \mathrm{~s}$ subunit. The $60 \mathrm{~s}$ subunit and $40 \mathrm{~s}$ subunit make up ribosomes that catalyse protein synthesis. RPL35 belongs to the L29 family of ribosomal proteins. These proteins of this family are usually associated with the activation of oncogenes and anti-oncogenes. However, there are few studies on RPL35. At present, studies on the identified diseases related to RPL35 mainly focus on Diamond-Blackfan anaemia (DBA) [44]. In addition, one study speculated that the expression of RPL35 could predict lymph node metastasis in patients with early-stage cervical carcinoma
[45]. A recent study reported that lncNB1 promotes neuroblastoma tumorigenesis by interacting with RPL35. This study also identified that RPL35 is a key factor for promoting E2F1 protein synthesis [46]. E2Fs are a classic group that regulate the occurrence of cancer and the cell cycle. Moreover, the GSEA results showed that the high expression of DDX10 is closely related to the cell cycle and E2F targets. Therefore, we speculated whether the E2F pathway is a key pathway in regulating CRC after DDX10 alternatively splices the mRNA of RPL35. To confirm our conjecture, we observed the relationship between DDX10, RPL35 and E2Fs in LinkedOmics. As expected, both DDX10 and RPL35 were closely related to E2F1, E2F2, E2F3, E2F4 and E2F5. Consequently, we believe that the E2F pathway is very likely to play an important role in the DDX10-RPL35-CRC regulatory pathway. Alternative splicing of mRNA is an important way to regulate gene expression in cancer development. As an RNA helicase, DDX10 is likely to participate 
in the splicing of the mRNA of RPL35. As we expected, high expression of DDX10 increased the incidence of AT (Alternate terminator) in mRNA of RPL35. Interestingly, through the TIMER database, we found that there was a strong correlation between the expression of DDX10 and the number of infiltrating immune cells, such as $\mathrm{CD} 4+\mathrm{T}$ cells, CD8 $+\mathrm{T}$ cells and macrophages, in COAD. Based on this discovery, we hypothesize that DDX10 may mediate the immune response in the development of CRC, which must be of great significance for the immunotherapy of CRC.

\section{Conclusion}

In summary, we determined that DDX10 is a novel oncogene that affects the mRNA expression of RPL35. Moreover, we speculate that RPL35 may be related to the E2F pathway and immune response in CRC. Our study showed that DDX10 is a potential therapeutic target for CRC and could have important significance for clinical research on CRC.

\section{Supplementary Information}

The online version contains supplementary material available at https://doi. org/10.1186/s12935-022-02478-1.

Additional file 1. DDX10 is a critical biomarker of colorectal cancer.

Additional file 2. Biological mechanism of DDX10.

Additional file 3: Table S1. The proteins selected by LC-MS/MS

\section{Acknowledgements}

Not applicable.

\section{Authors' contributions}

$X Y$ and $X Z$ conceptualized the project, designed research, supervised experiments, and wrote the manuscript. MJ, TH and ZL designed research, performed experiments, analyzed data, and wrote the manuscript. ZW, CZ, YJ and CG contributed to collate and analyze the new data and revised the manuscript. WZ contributed to modify the manuscript. All authors have read and approved the final manuscript.

\section{Funding}

This study was supported by the project of Suzhou Technology Bureau (No. SYS2018055), Suzhou health talent project (No. GSWS2020037), medical talent project of Jiangsu Commission of Health (No. QNRC2016873), the project of research and practice innovation plan for Postgraduates in Jiangsu Province (No. KYCX20-2721). All authors have read and approved the version of the manuscript submitted for publication.

\section{Availability of data and materials}

The data used in the current study are available from the corresponding author on reasonable request. The TCGA data of the gene expression RNAseq were from the database UCSC Xena. The GEO dataset (GSE74604) of the gene expression RNAseq were from GEO database.

\section{Declarations}

Ethics approval and consent to participate

The Ethics Committee of Soochow University.
Consent for publication

Not applicable.

\section{Competing interests}

The authors declare no competing interests.

\section{Author details}

'Department of General Surgery, The Second Affiliated Hospital of Soochow University, 1055 Sanxiang Road, Suzhou 215004, Jiangsu, China. ${ }^{2}$ Soochow University, 1 Shizi Street, Suzhou, China. ${ }^{3}$ Department of Radiotherapy, The Second Affiliated Hospital of Soochow University, 1055 Sanxiang Road, Suzhou 215004, Jiangsu, China.

Received: 15 September 2021 Accepted: 19 January 2022

Published online: 02 February 2022

\section{References}

1. Sung H, Ferlay J, Siegel RL, Laversanne M, Soerjomataram I, Jemal A, Bray F. Global cancer statistics 2020: GLOBOCAN estimates of incidence and mortality worldwide for 36 cancers in 185 countries. CA Cancer J Clin. 2021. https://doi.org/10.3322/caac.21660.

2. Dekker E, Tanis PJ, Vleugels JLA, Kasi PM, Wallace MB. Colorectal cancer. Lancet. 2019;394(10207):1467-80.

3. Bray F, Ferlay J, Soerjomataram I, Siegel RL, Torre LA, Jemal A. Global cancer statistics 2018: GLOBOCAN estimates of incidence and mortality worldwide for 36 cancers in 185 countries. CA Cancer J Clin. 2018:68(6):394-424

4. Singleton MR, Dillingham MS, Wigley DB. Structure and mechanism of helicases and nucleic acid translocases. Annu Rev Biochem. 2007;76:23-50.

5. Linder $P$, Jankowsky E. From unwinding to clamping - the DEAD box RNA helicase family. Nat Rev Mol Cell Biol. 2011;12(8):505-16.

6. Cordin O, Banroques J, Tanner NK, Linder P. The DEAD-box protein family of RNA helicases. Gene. 2006;367:17-37.

7. Germain DR, Graham K, Glubrecht DD, Hugh JC, Mackey JR, Godbout R. DEAD box 1: a novel and independent prognostic marker for early recurrence in breast cancer. Breast Cancer Res Treat. 2011;127(1):53-63.

8. Han C, Liu Y, Wan G, Choi HJ, Zhao L, Ivan C, He X, Sood AK, Zhang X, Lu X. The RNA-binding protein DDX1 promotes primary microRNA maturation and inhibits ovarian tumor progression. Cell Rep. 2014;8(5):1447-60.

9. Johanneson B, Chen D, Enroth S, Cui T, Gyllensten U. Systematic validation of hypothesis-driven candidate genes for cervical cancer in a genomewide association study. Carcinogenesis. 2014;35(9):2084-8.

10. Koenig AB, Barajas JM, Guerrero MJ, Ghoshal K. A comprehensive analysis of argonaute-CLIP data identifies novel, conserved and species-specific targets of miR-21 in human liver and hepatocellular carcinoma. Int J Mol Sci. 2018. https://doi.org/10.3390/ijms19030851.

11. Tanaka K, Ikeda N, Miyashita K, Nuriya H, Hara T. DEAD box protein DDX1 promotes colorectal tumorigenesis through transcriptional activation of the LGR5 gene. Cancer Sci. 2018;109(8):2479-89.

12. Guturi KK, Sarkar M, Bhowmik A, Das N, Ghosh MK. DEAD-box protein p68 is regulated by beta-catenin/transcription factor 4 to maintain a positive feedback loop in control of breast cancer progression. Breast Cancer Res. 2014;16(6):496.

13. Iyer RS, Nicol SM, Quinlan PR, Thompson AM, Meek DW, Fuller-Pace FV. The RNA helicase/transcriptional co-regulator, p68 (DDX5), stimulates expression of oncogenic protein kinase, Polo-like kinase-1 (PLK1), and is associated with elevated PLK1 levels in human breast cancers. Cell Cycle. 2014;13(9):1413-23.

14. Schulte I, Batty EM, Pole JC, Blood KA, Mo S, Cooke SL, Ng C, Howe KL, Chin SF, Brenton JD, et al. Structural analysis of the genome of breast cancer cell line ZR-75-30 identifies twelve expressed fusion genes. BMC Genom. 2012;13:719.

15. Dey H, Liu ZR. Phosphorylation of p68 RNA helicase by p38 MAP kinase contributes to colon cancer cells apoptosis induced by oxaliplatin. BMC Cell Biol. 2012;13:27.

16. Shin S, Rossow KL, Grande JP, Janknecht R. Involvement of RNA helicases p68 and p72 in colon cancer. Can Res. 2007;67(16):7572-8. 
17. Singh C, Haines GK, Talamonti MS, Radosevich JA. Expression of p68 in human colon cancer. Tumour Biol. 1995;16(5):281-9.

18. Felix RS, Colleoni GW, Caballero OL, Yamamoto M, Almeida MS, Andrade VC, Chauffaille Mde L, Silva WA Jr, Begnami MD, Soares FA, et al. SAGE analysis highlights the importance of p53csv, ddx5, mapkapk2 and ranbp2 to multiple myeloma tumorigenesis. Cancer Lett. 2009;278(1):41-8.

19. Mattioli M, Agnelli L, Fabris S, Baldini L, Morabito F, Bicciato S, Verdelli D, Intini D, Nobili L, Cro L, et al. Gene expression profiling of plasma cell dyscrasias reveals molecular patterns associated with distinct IGH translocations in multiple myeloma. Oncogene. 2005;24(15):2461-73.

20. Zhan F, Hardin J, Kordsmeier B, Bumm K, Zheng M, Tian E, Sanderson R, Yang Y, Wilson C, Zangari M, et al. Global gene expression profiling of multiple myeloma, monoclonal gammopathy of undetermined significance, and normal bone marrow plasma cells. Blood. 2002;99(5):1745-57.

21. Jiao X, Hooper SD, Djureinovic T, Larsson C, Warnberg F, Tellgren-Roth C, Botling J, Sjoblom T. Gene rearrangements in hormone receptor negative breast cancers revealed by mate pair sequencing. BMC Genom. 2013; 14:165.

22. Shi JH, Hao YJ. DDX10 overexpression predicts worse prognosis in osteosarcoma and its deletion prohibits cell activities modulated by MAPK pathway. Biochem Biophys Res Commun. 2019;510(4):525-9.

23. Gai M, Bo Q, Qi L. Epigenetic down-regulated DDX10 promotes cell proliferation through Akt/NF-kappaB pathway in ovarian cancer. Biochem Biophys Res Commun. 2016;469(4):1000-5.

24. Tang J, Chen H, Wong CC, Liu D, Li T, Wang X, Ji J, Sung JJ, Fang JY, Yu J. DEAD-box helicase 27 promotes colorectal cancer growth and metastasis and predicts poor survival in CRC patients. Oncogene. 2018;37(22):3006-21.

25. Ma J, Chang W, Zhang W. Relationship between the expression of DDX39 protein and prognosis of colorectal cancer. Chin J Gastrointest Surg. 2018;21(3):336-41.

26. Li M, Ma Y, Huang P, Du A, Yang X, Zhang S, Xing C, Liu F, Cao J. Lentiviral DDX46 knockdown inhibits growth and induces apoptosis in human colorectal cancer cells. Gene. 2015;560(2):237-44.

27. Wu DW, Lin PL, Wang L, Huang CC, Lee H. The YAP1/SIX2 axis is required for DDX3-mediated tumor aggressiveness and cetuximab resistance in KRAS-wild-type colorectal cancer. Theranostics. 2017;7(5):1114-32.

28. Kouyama Y, Masuda T, Fujii A, Ogawa Y, Sato K, Tobo T, Wakiyama H, Yoshikawa Y, Noda M, Tsuruda Y, et al. Oncogenic splicing abnormalities induced by DEAD-Box Helicase 56 amplification in colorectal cancer. Cancer Sci. 2019;110(10):3132-44.

29. Edgar R, Domrachev M, Lash AE. Gene Expression Omnibus: NCBI gene expression and hybridization array data repository. Nucleic Acids Res. 2002;30(1):207-10.

30. Tang Z, Li C, Kang B, Gao G, Li C, Zhang Z. GEPIA: a web server for cance and normal gene expression profiling and interactive analyses. Nucleic Acids Res. 2017;45(W1):W98-w102.

31. Vasaikar SV, Straub P, Wang J, Zhang B. LinkedOmics: analyzing multiomics data within and across 32 cancer types. Nucleic Acids Res. 2017;46(D1):D956-63.

32. Wang Y, Zhu L, Guo M, Sun G, Zhou K, Pang W, Cao D, Tang X, Meng X. Histone methyltransferase WHSC1 inhibits colorectal cancer cell apoptosis via targeting anti-apoptotic BCL2. Cell Death Discov. 2021;7(1):19.

33. Kanehisa M, Goto S. KEGG: kyoto encyclopedia of genes and genomes. Nucleic Acids Res. 2000;28(1):27-30.

34. Ashburner M, Ball CA, Blake JA, Botstein D, Butler H, Cherry JM, Davis AP, Dolinski K, Dwight SS, Eppig JT, et al. Gene ontology: tool for the unification of biology. The gene ontology consortium. Nat Genet. 2000;25(1):25-9.

35. Szklarczyk D, Gable AL, Lyon D, Junge A, Wyder S, Huerta-Cepas J, Simonovic M, Doncheva NT, Morris JH, Bork P, et al. STRING v11: protein-protein association networks with increased coverage, supporting functional discovery in genome-wide experimental datasets. Nucleic Acids Res. 2019;47(D1):D607-d613.

36. Li T, Fan J, Wang B, Traugh N, Chen Q, Liu JS, Li B, Liu XS. TIMER: a web server for comprehensive analysis of tumor-infiltrating immune Cells. Can Res. 2017;77(21):e108-10.

37. Li B, Severson E, Pignon JC, Zhao H, Li T, Novak J, Jiang P, Shen H, Aster JC, Rodig S, et al. Comprehensive analyses of tumor immunity: implications for cancer immunotherapy. Genome Biol. 2016;17(1):174.
38. van Voss MRH, Vesuna F, Bol GM, Meeldijk J, Raman A, Offerhaus GJ, Buerger $H$, Patel $A H$, van der Wall E, van Diest PJ, et al. Nuclear DDX3 expression predicts poor outcome in colorectal and breast cancer. OncoTargets Ther. 2017;10:3501-13.

39. Li XN, Wang ZJ, Ye CX, Zhao BC, Li ZL, Yang Y. RNA sequencing reveals the expression profiles of circRNA and indicates that circDDX17 acts as a tumor suppressor in colorectal cancer. J Exp Clin Cancer Res. 2018;37(1):325.

40. Zhang M, Weng W, Zhang Q, Wu Y, Ni S, Tan C, Xu M, Sun H, Liu C, Wei P, et al. The IncRNA NEAT1 activates Wnt/ $\beta$-catenin signaling and promotes colorectal cancer progression via interacting with DDX5. J Hematol Oncol. 2018;11(1):113.

41. Savitsky K, Ziv Y, Bar-Shira A, Gilad S, Tagle DA, Smith S, Uziel T, Sfez S, Nahmias J, Sartiel A, et al. A human gene (DDX10) encoding a putative DEAD-box RNA helicase at 11q22-q23. Genomics. 1996;33(2):199-206.

42. Arai Y, Hosoda F, Kobayashi H, Arai K, Hayashi Y, Kamada N, Kaneko Y, Ohki M. The inv(11)(p15q22) chromosome translocation of de novo and therapy-related myeloid malignancies results in fusion of the nucleoporin gene, NUP98, with the putative RNA helicase gene, DDX10. Blood. 1997:89(11):3936-44

43. Yassin ER, Abdul-Nabi AM, Takeda A, Yaseen NR. Effects of the NUP98DDX10 oncogene on primary human $\mathrm{CD}_{3}{ }^{+}$cells: role of a conserved helicase motif. Leukemia. 2010;24(5):1001-11.

44. Mirabello L, Khincha PP, Ellis SR, Giri N, Brodie S, Chandrasekharappa SC, Donovan FX, Zhou W, Hicks BD, Boland JF, et al. Novel and known ribosomal causes of Diamond-Blackfan anaemia identified through comprehensive genomic characterisation. J Med Genet. 2017;54(6):417-25.

45. Huang L, Zheng M, Zhou QM, Zhang MY, Jia WH, Yun JP, Wang HY. Identification of a gene-expression signature for predicting lymph node metastasis in patients with early stage cervical carcinoma. Cancer. 2011;117(15):3363-73.

46. Liu PY, Tee AE, Milazzo G, Hannan KM, Maag J, Mondal S, Atmadibrata B, Bartonicek N, Peng $\mathrm{H}, \mathrm{Ho} N$, et al. The long noncoding RNA IncNB1 promotes tumorigenesis by interacting with ribosomal protein RPL35. Nat Commun. 2019;10(1):5026.

\section{Publisher's Note}

Springer Nature remains neutral with regard to jurisdictional claims in published maps and institutional affiliations.

Ready to submit your research? Choose BMC and benefit from

- fast, convenient online submission

- thorough peer review by experienced researchers in your field

- rapid publication on acceptance

- support for research data, including large and complex data types

- gold Open Access which fosters wider collaboration and increased citations

- maximum visibility for your research: over $100 \mathrm{M}$ website views per year

At BMC, research is always in progress.

Learn more biomedcentral.com/submissions 